\title{
A Piezoelectric Device for Measurement and Power Harvesting Applications
}

\author{
M. Alves ${ }^{1}$, J.M. Dias Pereira ${ }^{1,3}$, J. M. Fonseca ${ }^{2}$, \\ ${ }^{1}$ ESTSetúbal-LabIM/IPS \\ R. Vale de Chaves, Estefanilha, 2910-761 Setúbal (Portugal) \\ Phone: +351265790000 \\ e-mail: mario.alves@estsetubal.ips.pt; dias.pereira@estsetubal.ips.pt \\ 2 Universidade Nova de Lisboa \\ Faculdade de Ciências e Tecnologia, 2829-516 Caparica \\ Phone: +351 212948380 \\ e-mail: jmrf@fct.unl.pt \\ 3 Instituto de Telecomunicações, Instituto Superior Técnico \\ Av. Rovisco Pais 1, 1049-001 Lisboa (Portugal) \\ Phone: +351 218418 454, Fax: +351218418472
}

\begin{abstract}
With the fast growth of wireless communications between nodes/sensor units, devices installed in remote places require power energy supply solutions to assure their functionally and data communication capabilities. For these applications energy harvesting takes place as a good solution, to increase the availability of energy, in opposition to the conventional systems of energy supply. Regenerative energy sources like thermoelectric, magnetic, piezoelectric, and/or renewable sources such as photovoltaic, wind, among others, allowed the development of different powering solutions for sensor units. The purpose of this work is to characterize a piezoelectric device to measure and capture mechanical vibrations from equipments, structures and piping vibrations, as well as from other sources. The study is carried out taking into account the power supply capabilities of piezoelectric devices as a function of the amplitude and frequency of the vibration stimulus, as well as, the electrical characteristics of the load circuit.
\end{abstract}

Keywords: Regenerative energy sources; energy harvesting; mechanical vibration energy; stand-alone systems; sensor applications.

\section{Introduction}

Nowadays, with electronics becoming smaller and requiring less power, the energy harvesting has began to be a topic of great importance to self-powering systems. The energy available in the surrounding environment of an application can be a viable 
option to allow the development of energy autonomous systems or to extend the autonomy of systems supplied by storage elements, usually batteries.

Different sources that capture the energy of the environment, like solar, wind, vibration and temperature gradients, have been target of recent developments to improve their performances and efficiency.

A type of environmental source as a global solution to supply a wide range of applications is not feasible, since the requisites of power/energy can be completely different in amplitude or frequency/time.

Many energy harvesting systems are oversized, either because there is no controller to manage and optimize the energy flow or because they are designed for worst-case scenarios [1]. Create a dedicated harvesting solution with low power electronic internal consumption, is crucial to improve the performance of energy harvesting systems.

The main problem of autonomous and self-powered devices is related with energy storage solutions. The energy that is not consumed by the system is generally requested when the source cannot deliver the amount of energy that guarantees the consumption needs. Two main storage solutions can be used: batteries and supercapacitors. The use of supercapacitors has the following advantages: life cycle, which is at least two orders of magnitude higher than the corresponding one of lead acid batteries; long operation time in a temperature range of $-40^{\circ} \mathrm{C}$ to $+60^{\circ} \mathrm{C}$; higher power capability; ability to deliver for short time intervals electrical energy at significantly higher power than batteries [2]. Batteries are usually indicated for long time supplies (energy) without requisites of instantaneous high consumption (power).

The scaled solution purposed here aims to verifying the possibility of suppling enough energy for monitorization of physic variables in instrumentation applications. This energy must also be sufficient to assure the wireless communication of data.

\section{A. Piezoelectric elements}

Piezoelectric material produces mechanical strain under the influence of an externally applied electrical field, and conversely produces electrical potential in response to an applied mechanical strain.

The goal of this work is to characterize a piezoelectric device to convert vibration mechanical energy to electrical voltage, not only as a measurement signal, but as lowpower energy source. In the instrumentation field it is possible to use this principle for energy harvesting in the measure of force and wind, and in some type of flowmeters (Figure 1). At the same time, they give the information about the wind speed, it is possible to use the range of voltage generated for supplying the integrated application. 


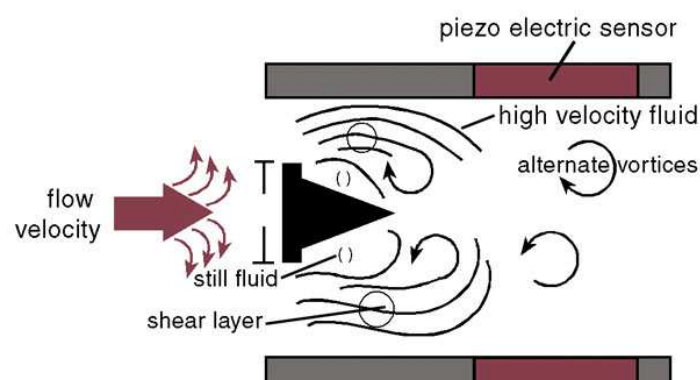

Fig. 1. Vortex flowmeter principle using piezoelectric elements

The measuring principle is based on development of Karmen Vortex shedding street in the wake of a body built into the pipeline. Vortex shedding creates alternate pressure conditions whose frequency is directly related with flow velocity, being the relationship, between these variables, given by:

$$
V=\frac{1}{S_{t h}} \times f \times d .
$$

$$
\begin{aligned}
& 0,2<\mathrm{S}_{\mathrm{th}}<0,21 \text { to } 300<\mathrm{R}<150000 \\
& \mathrm{Sth}-\text { Strouhal number } \\
& \mathrm{R}-\text { Reynolds number }
\end{aligned}
$$

\section{B. Cantilevered-beam Configuration}

Solutions for piezoelectric energy harvesting are typically based on a cantilevered-beam configuration (Figure 2). The piezoelectric beam is clamped at one end and the other end is allowed to oscillate freely in response to vibration normal to the flat surface of the beam, converting these vibrations to in-plane material strain. It is extremely important that the natural frequency of harvester configuration is tuned to match the vibration source. Vibration sources vary considerably in amplitude and dominant frequency. In most of environments, vibration is not made up of a single frequency but is typically made up of a set of main fundamental frequencies and associated harmonics. [3]

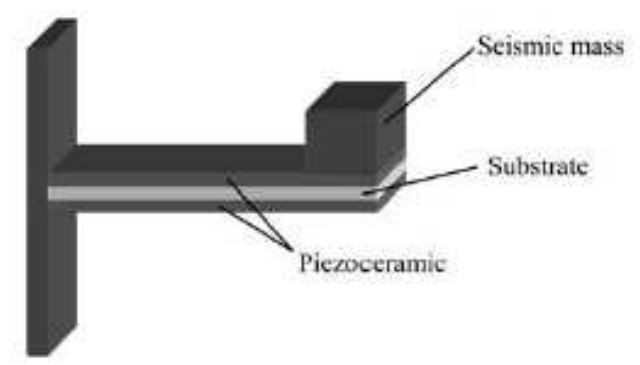

Fig. 2. Model of a cantilever-beam with piezoelectric ceramic layers

A cantilevered-beam piezoelectric energy harvester is a complex electromechanical system in which the electrical and mechanical loading of the beam are interrelated. 
The beam dimensions and tip mass determine the resonant frequency of the beam, which is tuned to match the dominant vibration frequency of its environment. These typically small vibrations are mechanically amplified.

A development and fabrication process of a cantilever-beam with a thin film piezoelectric power generator is presented by Jeon et al. [4]

\section{Main Contributions - Relationship to Cloud-based Engineering}

This work focuses a technological solution for power harvesting, with the aim at increasing the autonomy of low-power consumption applications. The present contribution intends to characterize a piezoelectric device as a solution to increase the energy sustainability and performance of instrumentation systems with wireless communication capabilities. This systems located in remote places have the ability to actualize information located in cloud data base computing systems. Particularly important for systems located in places where the viability of conventional energy supply is very expensive or technically difficult and it is crucial to minimize maintenance requirements. Figure 3 represents the block diagram of a sensing node with power harvesting and wireless communication capabilities.

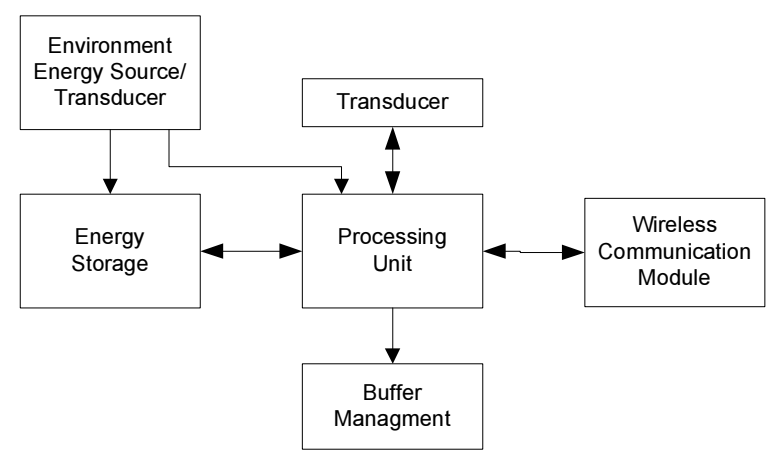

Fig. 3. Sensing node with power harvesting

\section{Methodology}

In this work two electrically isolated piezoelectric elements were used, whether independently or bridged for increased voltage (series configuration) or current output (parallel configuration). Series connection will double the open-circuit voltage compared to a single element, and the effective capacitance will be $1 / 2$ of the single capacitance. Parallel connection will double the current compared to a single element configuration, and doubling the effective capacitance. The option was to use the parallel connection in order to increase the power harvesting capability. However, the single element configuration was also tested and the results are presented as a reference for analyses facility. Each solution (single and parallel connection) was assembled in a cantilever-beam configuration.

The AC voltage at terminals of piezoelectric generator is in a first stage converted to DC voltage (Figure 4). The DC Voltage connected to a load permits defining the capability of generating power for instantaneous consumption, or for energy storage when connected to a supercapacitor. 
The open circuit voltage $\left(\mathrm{V}_{\mathrm{OC}}\right)$ and the short circuit current $\left(\mathrm{I}_{\mathrm{SC}}\right)$ are the two main parameters to start this system characterization (Figure 5). Furthermore, achieving the Maximum Power Point (MPP) in function of load for different amplitude and frequency vibration is important to optimize the performance of this type of energy harvesting. To maximize transfer efficiency, the load must be matched to the piezo's equivalent impedance. Theoretically, the piezo impedance at a given amplitude and frequency, as well as the load impedance, can be thought as a pair of simple (but unknown) impedances which make up an impedance divider. The power transfer between the two impedances is optimized when their resistive and reactive values match. This corresponds to the point at which the piezo's loaded voltage is equal to half its open-circuit voltage.

The simplicity of the solution that is proposed intends to represent an advantage in terms of internal power consumption.

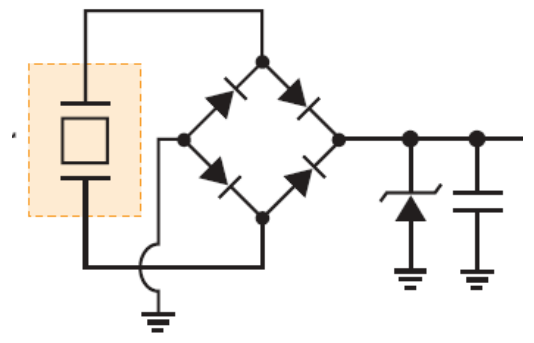

Fig. 4. Equivalent electric circuit AC-DC converter

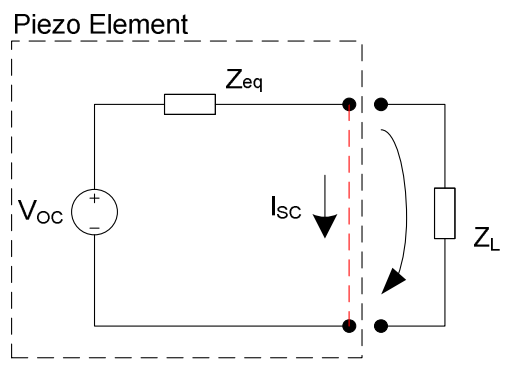

Fig. 5. Equivalent Thevenin circuit.

This circuit (Figure 4) is ideal for directly powering small sensors that can perform their function (e.g. record or transmit a measurement). It could also be used to extend battery life, of devices with their own battery/capacitor power source.

\section{Results and Critical View}

The cantilever-beam configuration tested with piezoelectric elements is strongly dependent on the environment frequency vibration, and not only on vibration amplitude. The system can be mounted in a large number of applications, but if for some reason the frequency of vibration change, the performance as harvester source will be very poor. This can be an important limitation for harvest energy from environments that do not have a constant vibration but depends of external factors like ambient conditions. However in some applications the vibration frequency is well know, like for instance, the structure of an AC motor working at $230 \mathrm{~V} / 50 \mathrm{~Hz}$. In this case the natural frequency of the piezoelectric harvester element must be tuned to $50 \mathrm{hz}$ to match the vibration source. In a cantilever configuration the frequency tuning is performed by adding mass to the end of the cantilever solution. The tuning mass can be adjusted until the vibration frequency and the natural frequency of the piezoelectric device are equal. The beam dimension associated with the mass is also determinant to achieve the resonant frequency of cantilever, and consequently the maximum power that will be possible to capture. In most applications it is very important, to characterize the frequency of the environment vibration in which the element will be operating. 
In this work the results were obtained for a established frequency of $50 \mathrm{~Hz}$. This frequency matches the vibration of an AC motor structure. We have also tested frequencies out of the tuned cantilever configuration, and it could confirm that, in this condition, the voltage level, and consequently the power harvesting, was extremely low. This short band wide of the cantilever with piezo elements is limitative as harvesting source and also as transducer for variables measurement. One solution to solve this problem, could be using different cantilever assembled in parallel each one tuned at a different frequency.

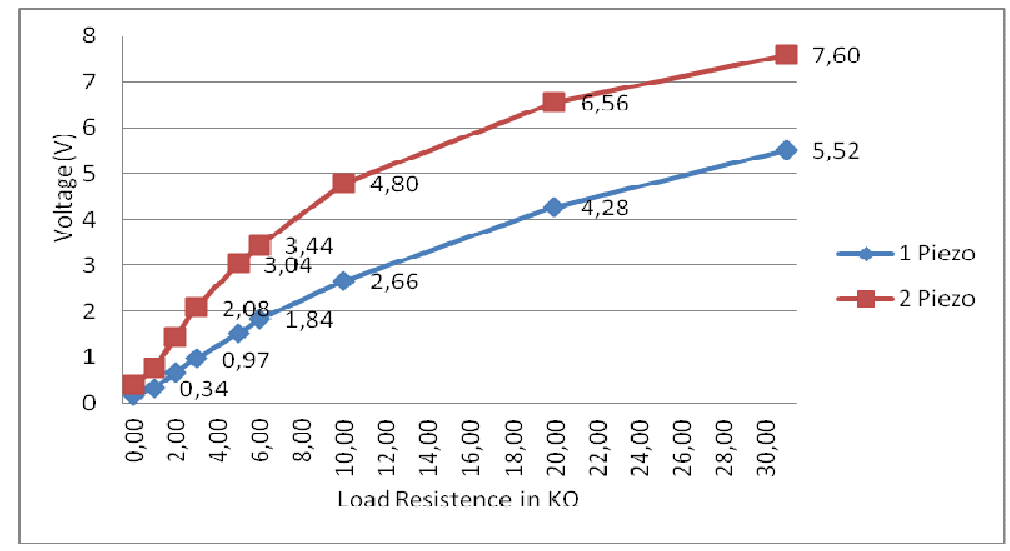

Fig. 6. Output Voltage in function of Load value

The DC output voltage (Vout) shown in figure 6 is lower than expected, due to the voltage drop in each diode $(0,7 \mathrm{~V})$. Since low power consumption is involved, it is possible the use of Schottky diodes with voltage drops as low as $0,15 \mathrm{~V}$.

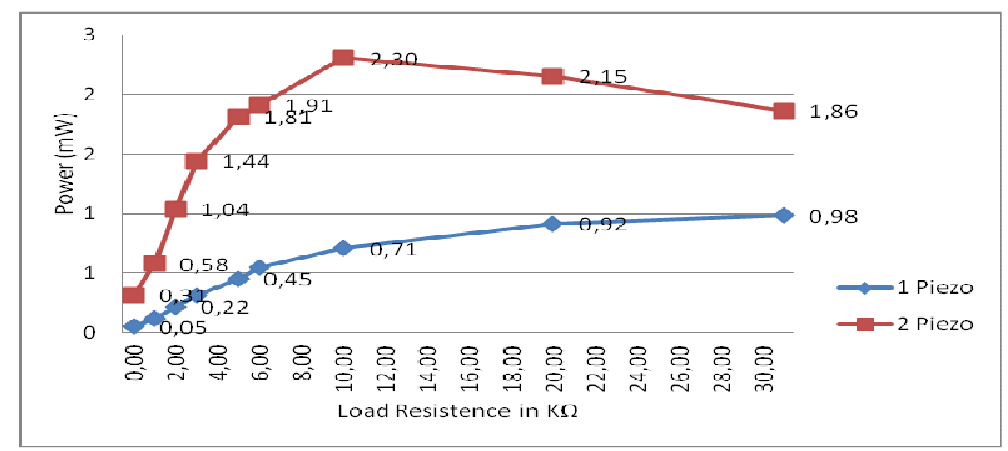

Fig. 7. Power harvesting in function of Load

Figure 7 shows the power harvesting behavior as a function of different load impedance, keeping the amplitude vibration constant. A clear difference was observed depending on the assembly in beam of a single or double piezoelectric elements. The power harvesting increased 3 fold for the majority of loads tested and MPP it was obtained for lower load impedances. This is significant to determine the load connected and also for the level and period necessary for charging the storage element. By comparing figure 6 and 7 it is possible to conclude that the 
power increase obtained corresponds to only a small increase in the output voltage. This is mainly due to the increase in current owed to parallel configuration.

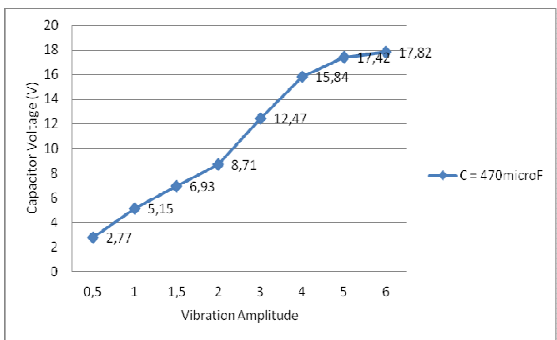

Fig.8. Capacitor Voltage in function of Vibration Amplitude with $\mathrm{f}=50 \mathrm{~Hz}$

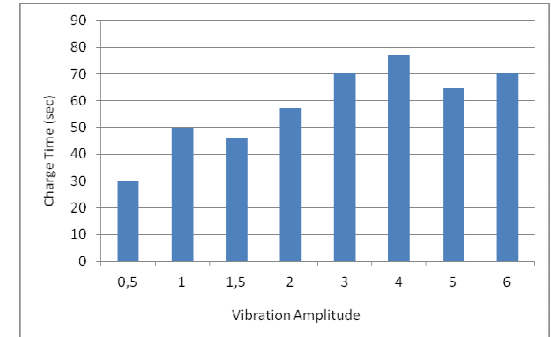

Fig.9. Time of capacitor charging in function of Vibration Amplitude with $\mathrm{f}=50 \mathrm{~Hz}$

The results presented in figure 8 and 9 correspond to a established frequency of $50 \mathrm{~Hz}$ and 2 piezoelectric elements connected in parallel. The piezoelectric elements output was rectified and connected to a capacitive storage of $470 \mu \mathrm{F}$. The voltage at the terminals of the capacitor and the time necessary for charging are represented for different amplitudes of vibration. We can observe that the storage element subjected to the highest vibration amplitude could charge 6 times higher level of voltage during only the double-time to charge. Tests with capacitors of higher value and the same configuration of two piezo elements were done as well. Lower levels of voltage and higher period of charging were observed in this case, showing that the power harvesting is very low for storage elements with higher capacity.

Taken together these results allowed to verify that good levels of power harvesting are possible when several piezoelectric elements assembled in parallel, which may have a promising application.

\section{Conclusion and further work}

The piezoelectric materials are very sensitive to the surrounding vibration, producing easily very good levels of voltage. This characteristic is undoubtedly very important for vibration measurement. The use of these elements for power harvesting are however limited by the fact that the levels of voltage are not followed by good values of power supplies capabilities, exhibiting low current values. One hypothesis to solve the problem is the use of several piezoelectric elements connected in parallel. By using this method the power harvesting by piezoelectric elements can be a promising solution for low power consumption applications. Since this method can only be used in application where the environment vibration frequency has a low bandwidth, the best approach is to start by characterizing the vibration variation and the levels of power harvesting required.

New type of materials and ceramics are now being tested and developed to improve the piezoelectric characteristics. This will enable new values and facilities for power harvesting and measurement solutions and will probably greatly improve the efficiency and application of this method. 


\section{References}

1. Kimball, J.W., Kuhn, B.T., Balog, R.S.: A System Design Approach for Unattended Solar Energy Harvesting Supply. IEEE Transactions on Power Electronics, Vol. 24, No. 3-4, pp. 952-962, Mar.-Apr. (2009)

2. Obreja, V.V.N.: On the Performance of Commercial Supercapacitors as Storage Devices for Renewable Electrical Energy Sources. in: ICREPQ’07, Sevilla, Spain, Mar. 2007.

3. Gilbert, James M., Baloucchi, Farooq: Comparison of Energy Harvesting Systems for Wireless Sensor Networks. in: International Journal of Automation and Computing, pp. 334-347, October 2008.

4. Y.B. Jeon, R. Sood, J.-h- Jeong, S.-G. Kim: MEMS power generator with transverse mode thin film PZT. in: Sensors and Actuators A Physical, pp. 16-22, 2005. 